\title{
Relación entre el Estilo Educativo parental y el nivel de adaptación de menores en riesgo social
}

\author{
Josefina Rodríguez Góngora ${ }^{1}$, María del Carmen Pérez-Fuentes ${ }^{2}$ y José Jesús \\ Gázquez Linares ${ }^{2}$ \\ ${ }^{1}$ Excma. Diputación Provincial de Almería; ${ }^{2}$ Universidad de Almería (España)
}

\begin{abstract}
Fruto del aumento de menores con comportamiento disruptivo y problemas de adaptación, aparecen diferentes investigaciones centradas en el análisis de los aspectos que influyen en su aparición. Así, el objetivo central de este trabajo es analizar la relación entre el Estilo Educativo parental y el Nivel de Adaptación (Personal, Familiar, Escolar y Social) de menores en riesgo social. Para ello, se realizó un estudio descriptivo y de diseño transversal, cuya muestra estuvo formada por 17 familias, seleccionadas en función de la edad de los/las hijos/as (entre 11 y 15 años), con un total de 31 padres/madres y 17 menores. Los resultados nos muestran niveles bajos de adaptación personal, escolar e insatisfacción con el ambiente familiar y discrepancias educativas, tanto en la percepción de los menores, como en los estilos de los progenitores. La Inadaptación Escolar de los menores se relaciona con un Estilo Educativo Permisivo de su madre; mientras que, los menores se sienten insatisfechos con sus hermanos y muestran Inadaptación Personal, cuando la madre ejerce un Estilo Educativo Democrático. Por otro lado, cuando los padres ejercen un Estilo Educativo Autoritario se encuentra una mayor presencia de Inadaptación Personal y Social.
\end{abstract}

Palabras clave: Bienestar social, adaptación personal, social, familiar y escolar, menores en riesgo social, estilo educativo.

Relation between parental Educational Style and level of adaptation of minors at social risk. As a result of the growing number of minors with disruptive behavior and adaptation problems, more research is being published focusing on the analysis of aspects that influence the onset of these problems. Thus, the main goal of this work is to analyze the relation between parental Educational Style and the Level of Adaptation (Personal, Family, and Social) in minors at social risk. For this purpose, a descriptive, cross-sectional study was carried out, with a sample made up of 17 families, selected as a function of the children's age (between 11 and 15 years), with a total of 31 fathers/mothers and 17 minors. The results show low levels of personal and school adaptation, dissatisfaction with the family environment, and educational discrepancies, both in the minors' perception and in the parents' styles. Minors' school maladjustment is related to a Permissive Educational Style in the mother, whereas the minors were dissatisfied with their siblings and displayed Personal Maladjustment when the mother employed a Democratic Educational Style. However, when the parents employ an Authoritarian Educational Style, a greater presence of Personal and Social Maladjustment is observed in their children.

Key words: Social well-being; personal, social, family, and school adaptation, minors at social risk, educational style.

Correspondencia: José Jesús Gázquez Linares. Departamento de Psicología. Universidad de Almería. Ctra. Sacramento, s/n. C.P.: 04120. La Cañada de San Urbano, Almería (España). E-mail: jlinares@ual.es 
La condición educativa de la familia no ofrece margen de duda, habiéndose establecido en la sociedad, a lo largo de los años, la concienciación sobre la importancia del papel que juegan los progenitores en el desarrollo y progreso psicoeducativo de sus hijos/as (Martínez, 2004). Así, algunos autores han destacado la gran influencia ejercida por el temperamento de los menores en los estilos de crianza, con sus diferencias individuales, siendo esto un condicionante importante en el ajuste social del menor (Ato, Galián y Huéscar, 2007). De igual forma, encontramos que un óptimo ajuste psicológico, así como, una buena competencia personal y comportamental, están relacionados de forma directa con el autoconcepto (Fuentes, García, Gracia y Lila, 2011). Aunque, no se observa una relación significativa entre el uso de estrategias de aprendizaje y niveles altos de autoestima, sí existe relación entre ésta y la planificación de respuesta, con puntuaciones más altas en las subescalas familiar, social y corporal (Gázquez, Pérez-Fuentes, Ruiz, Miras y Vicente, 2006).

El nivel cultural determina las expectativas, valores y actitudes que las familias tienen respecto a la educación de los menores. El nivel cultural familiar ejerce mayor influencia, en la motivación de logro para los menores, que el nivel económico (Llorente, 1990). Así pues, los alumnos de clase social baja presentan un peor uso de las estrategias metacognitivas que los alumnos de clase social alta (Marchesi y Martín, 2002), con incidencia directa en el rendimiento académico (Buote, 2001; Castejón y Pérez, 1998; Caplan, Henderson y Fleming, 2002; Martínez-Otero, 1997).

Así mismo, las familias incluidas en el Programa de Tratamiento a familias con menores (Dirección General de Infancia y Familia, 2007) presentan características específicas que difieren de la población normal en aspectos como son: bajo estatus socio-económico, bajo nivel cultural y presencia de factores de riesgo e indicadores de maltrato infantil. Igualmente, los escolares con pobre adaptación escolar presentan condiciones familiares como: estructura familiar difusa, carencia de interacciones y actividad recreativa, falta de identificación de los menores con figuras adultas de la familia, baja autoestima, relaciones conflictivas dentro del hogar, predominio de castigos físicos o indiferencia sobre otros métodos disciplinarios (Platone, 2007). Así, un alto índice de factores de riesgo pueden precipitar en situaciones críticas de maltrato, y factores como la socialización de riesgo, la búsqueda de sensaciones y escasa comunicación y déficit de toma de perspectiva, pueden desencadenar en conducta disocial (De la Rubia y Ortiz, 2011).

Por otro lado, las prácticas educativas paternas se relacionan con las conductas manifiestas por los adolescentes (García, Cerezo, De la Torre, Carpio y Casanova, 2011) mostrando que las prácticas negativas se relacionan positivamente con los problemas internalizantes y externalizantes, mientras que las prácticas positivas se relacionan positivamente con los problemas externalizantes, observándose diferencias en las variables predictoras en chicos y en chicas, con mayor predicción en el caso de los 
problemas externalizantes. Los jóvenes perciben las prácticas educativas de las madres más positivas que las de los padres, las chicas parecen más sensibles a las diferencias entre ambos progenitores. Al realizar una comparativa de las percepciones que niños y niñas tienen del estilo educativo (EE) materno y del paterno, encontramos que a pesar de la gran concordancia percibida entre ambos estilos, conceden puntuaciones más altas a sus madres en cada una de las dimensiones del EE (Oliva, Parra, Sánchez y López, 2007). Por tanto, el EE de los padres influye de manera directa en el proceso educativo de los menores, así como, en las relaciones familia y escuela, sin olvidar otro elemento importante como es el profesorado (Santiago, Otero, Castro y Villardefrancos, 2008). Un clima familiar positivo favorece la formación de sujetos adaptados, maduros, estables e integrados, mientras que, un clima familiar desfavorable promueve la inadaptación, inmadurez, desequilibrio e inseguridad (Rodríguez, 1986), siendo los Estilos Educativos (EEs) paternos elementos clave de la socialización familiar (Torio, Peña e Inda, 2008).

Algunos de los EEs: Autoritario, Sobreprotector y Permisivo tienen efectos perjudiciales hacia los menores (Ríos, 1973). El autoritarismo provoca ansiedad, con alteración en factores de personalidad, autoconcepto social y escolar, motivación, relación social, etc. Igualmente, la sobreprotección tiene efectos y consecuencias también negativas para el niño/a, como sentimiento de culpabilidad, retraso afectivo, retraso escolar, infantilismo, timidez y problemas sociales, y el EE permisivo implica dejar al margen toda influencia del medio social en la generación de comportamientos. El exceso de autoritarismo por parte de padres/tutores no es considerado por personas mayores como el origen de conductas violentas, al igual que el exceso de libertad o falta de autoridad están entre los elementos que más influyen en su presencia (Gázquez, Pérez-Fuentes y Carrión, 2009). Son, por tanto, EEs que se relacionan con la inadaptación familiar (Rollán et al., 2011). Los EEs autoritarios y permisivos influyen de manera negativa sobre la motivación y realización de tareas escolares (Fernández y Salvador, 1994). Los menores cuyos padres son clasificados como democráticos y como permisivos, utilizan en mayor medida estrategias de aprendizaje autorregulado si los comparamos con los estudiantes cuyos padres son clasificados como autoritarios y negligentes, constatándose como este aprendizaje autorregulado incide sobre el logro académico de los menores (Cerezo, Casanova, De la Torre y Carpio, 2011). Por todo esto, podemos deducir la existencia de relación positiva del EE autoritario con la inadaptación personal y social en niños y niñas (López-Soler, Puerto, López-Pina y Prieto, 2009) y bajo rendimiento escolar de los hijos (Steinberg, 1989; Lamborn, 1991; Domínguez y Carton, 1997 y Fernández y Salvador, 1994). En cambio, los menores con padres con utilización de EE democrático tienen mayor competencia social y autoconfianza que los menores con padres con utilización de EE Autoritario (García-Linares, Pelegrina y Lendínez, 2002). El EE Democrático parece favorecer el nivel de ajuste de los menores, manifestando durante la infancia un estado emocional 
estable y alegre con elevado nivel de autoestima y autoconcepto (Alonso y Román, 2005). Por tanto, el EE Democrático parece influir de manera positiva en el nivel de adaptación de los menores, siendo los otros EEs aquellos que producen un nivel de inadaptación mayor en los mismos; dicho EE es el más adecuado ya que facilita una mejor adaptación (Steinberg, 2001), por lo que un clima familiar positivo favorece la formación de sujetos adaptados, maduros, estables e integrados (Rodríguez, 1986) y con mayor nivel de ajuste psicosocial de los/las hijos/as (García y Gracia, 2010). Del mismo modo, una mayor capacidad para reconocer emociones de los demás lleva asociada una mejor relación y menor tensión en las relaciones sociales con los iguales y con los propios padres (Salguero et al., 2011).

Como se ha visto, los adolescentes que perciben a sus padres como democráticos y permisivos obtienen altos niveles de socialización y adaptación, mientras que aquellos que los perciben como autoritarios o indiferentes obtienen niveles bajos en dichos factores, siendo la alta cohesión, expresividad, participación en actividades culturales-intelectuales, organización y bajos niveles de conflicto, así como, creatividad y por tanto quizás también la inteligencia, ya que están relacionadas (Ferreira, Martínez y Guisande, 2009), son las dimensiones que más favorecen una adecuada adaptación y socialización (Pichardo, 1998). Así pues, el EE Democrático favorece el nivel de ajuste de los menores (Alonso y Román, 2005) y la competencia social y autoconfianza (García-Linares et al., 2002) siendo dicho estilo el más adecuado ya que facilita una mejor adaptación familiar de los/las hijos/as (Rollán et al., 2011; Steinberg, 2001).

Por tanto, podemos llegar a la conclusión que el estilo óptimo de educación y crianza de los/las hijos/as es el democrático y el establecimiento temprano de prácticas efectivas es una tarea importante, tanto para el ajuste social del menor como para su éxito académico. La adopción de un estilo parental que ejerce su autoridad con responsabilidad, afecto y flexibilidad es el más beneficioso para el crecimiento emocional, moral e intelectual del menor (Bornstein y Bornstein, 2010). Por ello, el objetivo de nuestro trabajo es analizar y describir la relación entre el EE parental y el nivel de adaptación, de menores en situación de vulnerabilidad social.

\section{MÉTODO}

\section{Participantes}

La muestra utilizada es seleccionada de entre las familias incluidas en el Programa de Tratamiento a familias con menores (Dirección General de Infancia y Familia, 2007) distribuidas en diferentes municipios menores de 20.000 habitantes, pertenecientes a las zonas de trabajo social de Alpujarra y Poniente, dependientes de la Excma. Diputación Provincial de Almería, y están en intervención por parte del Equipo de Tratamiento Familiar de dichas zonas. Está formada por 17 menores pertenecientes a 
17 familias (tres de ellas monoparentales), con un total de 31 padres, de los que 15 (48.8\%) son varones con edades entre 28 y 51 años $(M=37 ; D T=7.26)$, y 16 son mujeres con edades entre 27 y $49(M=35 ; D T=6.78)$ (Tabla 1). Los menores tienen edades comprendidas entre 11 y 15 años, de los que 10 (58.8\%) son niños $(M=12.1 ; D T=1.19)$ y $7(41.2 \%)$ son niñas $(M=13.28 ; D T=1.49)$, distribuidos de la siguiente manera: de 11 años el $23.6 \%$, de 12 el $35.4 \%$, de 13 el $17.7 \%$, de 14 el $5.9 \%$ y de 15 el $17.7 \%$ (Tabla 2). Los menores cursan estudios desde $6^{\circ}$ de Educación Primaria a $2^{\circ}$ de Educación Secundaria Obligatoria.

Tabla 1. Distribución de los padres y madres por edad y género

\begin{tabular}{crrr}
\hline & Padres & Madres & Total \\
\hline Edad & $N(\%)$ & $N(\%)$ & $N(\%)$ \\
\hline$\leq 32$ & $5(16.1)$ & $8(25.6)$ & $13(42)$ \\
$33-36$ & $2(6.4)$ & $3(9.6)$ & $5(16.1)$ \\
$37-40$ & $3(9.6)$ & $2(6.4)$ & $5(16.1)$ \\
$41-44$ & $2(6.4)$ & $1(3.2)$ & $3(9.7)$ \\
$\geq 45$ & $3(9.6)$ & $2(6.4)$ & $5(16.1)$ \\
\hline
\end{tabular}

Tabla 2. Distribución de los menores por edad y género

\begin{tabular}{crrr}
\hline & Niños & Niñas & Total \\
\hline Edad & $N(\%)$ & $N(\%)$ & $N(\%)$ \\
\hline 11 & $3(17.7)$ & $1(5.9)$ & $4(23.6)$ \\
12 & $5(29.5)$ & $1(5.9)$ & $6(35.4)$ \\
13 & $1(5.9)$ & $2(11.8)$ & $3(17.7)$ \\
14 & $0(0)$ & $1(5.9)$ & $1(5.9)$ \\
15 & $1(5.8)$ & $2(11.8)$ & $3(17.7)$ \\
\hline
\end{tabular}

\section{Instrumentos}

Los instrumentos de medida utilizados han sido:

Test Autoevaluativo Multifactorial de Adaptación Infantil (TAMAI; Hernández, 1990). Consta de 175 proposiciones a través de las cuales se evalúan 4 escalas de inadaptación (Inadaptación Personal (IP), Inadaptación Escolar (IE), Inadaptación Social (IS), Inadaptación Familiar (IF)); el EE percibido (el EE Asistencial-personalizado, EE Permisivo, EE Restrictivo, Punitivo, Preocupado y Perfeccionista hostil, EE Perfeccionista hipernómico); así como, la existencia de Discrepancia educativa entre los progenitores.

Perfil de Estilos Educativos (PEE; Magaz y García, 1998). Tiene por finalidad el valorar de manera cuantitativa y cualitativa las actitudes y valores ante la educación de los/las hijos/as, según EE utilizado (Democrático, Autoritario, Permisivo y Sobreprotector). El PEE, es un cuestionario en forma de escala dicotómica, en la cual se ofrecen al sujeto 48 frases que expresan de manera afirmativa unas ideas, creencias, actitudes y emociones (asociadas a valores) sobre la educación de sus hijos/as. Las variables que evalúa, son: actitudes y valores de sobreprotección educativa, de inhibición educativa, de punición educativa y de aserción educativa. 
Debido a la no concordancia en la nomenclatura de los distintos estilos educativos medidos por ambas escalas, se han emparejado según se muestra en la tabla 3.

Tabla 3. Estilos educativos según Test Autoevaluativo de Adaptación Infantil (TAMAI; Hernández, 1990) y Perfil de Estilos Educativos (PEE; Magaz y García, 1998)

\begin{tabular}{ll}
\hline $\begin{array}{c}\text { Test Autoevaluativo de Adaptación Infantil } \\
\text { (TAMAI; Hernández, 1990) }\end{array}$ & \multicolumn{1}{c}{$\begin{array}{c}\text { Perfil de Estilos Educativos } \\
\text { (PEE; Magaz y García, 1998) }\end{array}$} \\
\hline Estilo educativo asistencial-personalizado & Estilo educativo democrático \\
\hline Estilo educativo permisivo & Estilo educativo permisivo \\
\hline $\begin{array}{l}\text { Estilo educativo restrictivo, punitivo, despreocupado y } \\
\text { perfeccionista hostil }\end{array}$ & Estilo educativo autoritario \\
\hline Estilo educativo perfeccionista hipernómico & Estilo educativo sobreprotector \\
\hline Fuente: Elaboración propia. &
\end{tabular}

\section{Procedimiento}

Las familias han sido informadas sobre las condiciones del estudio, autorizando a la utilización de los datos con los fines expuestos para la investigación, de conformidad con la Ley de Protección de Datos de carácter personal de 13 de diciembre de 1999.

Para la investigación se han aplicado dos cuestionarios, el Test Autoevaluativo Multifactorial de Adaptación Infantil (TAMAI; Hernández, 1990) y el Perfil de Estilos Educativos (PEE; Magaz y García, 1998), a los menores y progenitores respectivamente.

En primer lugar, se aplicó el Test Autoevaluativo Multifactorial de Adaptación Infantil (TAMAI; Hernández, 1990) a todos los menores en el ámbito educativo y de manera individual. Previamente se habló con los tutores de los menores en los diferentes Centros Educativos, a los que se les facilitó la autorización firmada por los progenitores para poder administrar el cuestionario. En el caso de los/las niños/as fue necesaria la presencia del evaluador, para facilitar la correcta implementación de la prueba, ofreciendo (de manera individual) apoyo y ayuda, para la correcta comprensión de todas las preguntas del cuestionario.

Posteriormente se procedió a aplicar el Perfil de Estilos Educativos (PEE; Magaz y García, 1998) a cada uno de los progenitores de forma individualizada, en los diferentes Centros de Servicios Sociales de la zona. La administración del mismo se realizó de manera individual, en presencia del evaluador, con la finalidad de aclarar y resolver dudas respecto a su cumplimentación, ya que el nivel educativo de algunos de los/las padres/madres es muy bajo, proporcionando a todos los/las padres/madres las explicaciones necesarias para la adecuada comprensión de todos los ítems.

Tras la cumplimentación de todos los cuestionarios, elaborados por parte de padres/madres y menores, se procedió a la realización del análisis estadístico de las distintas variables. 


\section{Análisis de datos/Diseño}

Se realizó un estudio descriptivo y de carácter transversal, al igual que en otros estudios (Ferreira, Martínez y Guisande, 2009; Goñi e Infante, 2010). Para realizar el procesamiento de datos y análisis estadístico se utilizó el software SPSS-19, que nos permitió analizar y trabajar estadísticamente los resultados. Dicho análisis ha consistido en calcular los estadísticos descriptivos (media, puntuación máxima, mínima y desviación típica) de cada una de las variables utilizadas en el estudio, además se realizaron pruebas $t$ de Student, $\chi^{2}$, Anova de un factor y Correlaciones.

\section{RESULTADOS}

Tabla 4. Estadísticos descriptivos (media, desviación típica, mínimo, máximo) de cada uno de los factores del

\begin{tabular}{lccc}
\multicolumn{4}{c}{ Test Autoevaluativo de Adaptación Infantil (TAMAI; Hernández, 1990) } \\
\hline \multicolumn{1}{c}{ Variable } & $N$ & Media & $D T$ \\
\hline Inadaptación General & 17 & 41.88 & 14.31 \\
\hline Inadaptación Personal & 17 & 14.47 & 5.97 \\
\hline Inadaptación Escolar & 17 & 15.29 & 5.85 \\
\hline Inadaptación Social & 17 & 12.12 & 4.31 \\
\hline Insatisfacción Ambiente Familiar & 17 & 1.35 & 1.32 \\
\hline Insatisfacción Hermanos & 17 & 1.88 & 1.79 \\
\hline Estilo Educativo del Padre & & & \\
\hline Democrático & 17 & 7.73 & 3.91 \\
\hline Sobreprotector & 17 & 0.33 & 0.61 \\
\hline Permisivo & 17 & 0.80 & 0.56 \\
\hline Autoritario & 17 & 3.80 & 2.08 \\
\hline Estilo Educativo de la Madre & & & \\
\hline Democrático & 17 & 9.19 & 3.92 \\
\hline Sobreprotector & 17 & 0.94 & 0.93 \\
\hline Permisivo & 17 & 1.06 & 0.44 \\
\hline Autoritario & 17 & 1.31 & 1.53 \\
\hline Discrepancia Educativa & 17 & 2.90 & 2.01 \\
\hline & & &
\end{tabular}

En cuanto al nivel de adaptación de los menores, se obtienen puntuaciones Casi Altas en nivel de Inadaptación General (M=41.88; DT=14.31), en Inadaptación Escolar (M=15.29; DT=5.85), Insatisfacción con Ambiente Familiar $(M=1.35 ; D T=1.32)$ y en Insatisfacción con Hermanos $(M=1.88 ; D T=1.79)$ y puntuaciones Altas en Inadaptación Personal ( $M=14.47 ; D T=5.97)$ (Tabla 3).

En relación al EE, se evidencia que los menores perciben que los padres presentan en mayor proporción un EE Autoritario con puntuaciones Muy Altas $(M=3.80$; $D T=2.08)$ seguido del Permisivo con puntuaciones Casi Altas $(M=0.80 ; D T=0.56)$. En referencia al EE materno, se aprecian puntuaciones Altas tanto para en EE Autoritario $(M=1.31 ; D T=2.01)$ como para el Permisivo $(M=1.06 ; D T=0.44)$. También aparecen puntuaciones Casi Altas en Discrepancia Educativa de los progenitores ( $M=2.9$; $D T=2.01$ ) (Tabla 4). 
Se analizan tanto el grado inadaptación como de insatisfacción de los menores en base al género de éstos y encontramos que en todas las variables de inadaptación los niños presentan valores más elevados que las niñas estando enmarcados en la mismas categorías, excepto en Inadaptación Escolar, donde los niños presentan puntuaciones Altas $(M=17.10 ; D T=5.15)$ y las niñas puntuaciones Casi Altas $(M=12.71 ; D T=17.10)$. Por el contrario, en las variables de insatisfacción son las niñas las que mayores índices presentan, aun encontrándose dentro de las mismas categorías que los niños. Al realizar el análisis correlacional, no se observan diferencias significativas en ninguno de los parámetros de inadaptación, así como tampoco en la Insatisfacción con el Ambiente Familiar e Insatisfacción con los Hermanos (Tabla 5).

Tabla 5. Diferencias de género e inadaptación

\begin{tabular}{|c|c|c|c|c|c|c|}
\hline & Género & $N$ & Media & $D T$ & $t$ & Sig. \\
\hline \multirow{2}{*}{ Inadaptación General } & Hombre & 10 & 45.80 & 13.55 & \multirow{2}{*}{1.38} & \multirow{2}{*}{.18} \\
\hline & Mujer & 7 & 36.29 & 14.44 & & \\
\hline \multirow{2}{*}{ Inadaptación Personal } & Hombre & 10 & 15.50 & 6.35 & \multirow{2}{*}{0.84} & \multirow{2}{*}{.41} \\
\hline & Mujer & 7 & 13.00 & 5.51 & & \\
\hline \multirow{2}{*}{ Inadaptación Escolar } & Hombre & 10 & 17.10 & 5.15 & \multirow{2}{*}{1.59} & \multirow{2}{*}{.13} \\
\hline & Mujer & 7 & 12.71 & 6.18 & & \\
\hline \multirow{2}{*}{ Inadaptación Social } & Hombre & 10 & 13.20 & 4.07 & \multirow{2}{*}{1.26} & \multirow{2}{*}{.23} \\
\hline & Mujer & 7 & 10.57 & 4.46 & & \\
\hline \multirow{2}{*}{ Insatisfacción Ambiente Familiar } & Hombre & 10 & 1.20 & 1.31 & \multirow{2}{*}{-0.56} & \multirow{2}{*}{.58} \\
\hline & Mujer & 7 & 1.57 & 1.40 & & \\
\hline \multirow{2}{*}{ Insatisfacción con Hermanos } & Hombre & 10 & 1.80 & 1.75 & \multirow{2}{*}{-0.22} & \multirow{2}{*}{.83} \\
\hline & Mujer & 7 & 2.00 & 2.00 & & \\
\hline
\end{tabular}

Tabla 6. Distribución del EE en los progenitores, percibido por los menores según Test Autoevaluativo de Adaptación Infantil (TAMAI; Hernández, 1990)

\begin{tabular}{|c|c|c|c|c|c|c|c|}
\hline & & Género & $N$ & $M$ & $D T$ & $t$ & Sig. \\
\hline \multirow{8}{*}{ EE del padre } & \multirow{2}{*}{ D } & Hombre & 9 & 7.78 & 4.11 & \multirow{2}{*}{0.05} & \multirow{2}{*}{.96} \\
\hline & & Mujer & 6 & 7.67 & 3.98 & & \\
\hline & \multirow{2}{*}{$\mathrm{P}$} & Hombre & 9 & 0.44 & 0.73 & \multirow{2}{*}{0.85} & \multirow{2}{*}{.41} \\
\hline & & Mujer & 6 & 0.17 & 0.40 & & \\
\hline & \multirow{2}{*}{$\mathrm{S}$} & Hombre & 9 & 0.56 & 0.52 & \multirow{2}{*}{-2.39} & \multirow{2}{*}{$.03^{*}$} \\
\hline & & Mujer & 6 & 1.17 & 0.40 & & \\
\hline & \multirow{2}{*}{ A } & Hombre & 9 & 3.56 & 2.55 & \multirow{2}{*}{0.40} & \multirow{2}{*}{.69} \\
\hline & & Mujer & 6 & 4.17 & 3.37 & & \\
\hline \multirow{8}{*}{ EE de la madre } & \multirow{2}{*}{$\mathrm{D}$} & Hombre & 9 & 9.33 & 4.03 & \multirow{2}{*}{0.16} & \multirow{2}{*}{.87} \\
\hline & & Mujer & 7 & 9.00 & 4.08 & & \\
\hline & \multirow{2}{*}{$\mathrm{P}$} & Hombre & 9 & 0.89 & .093 & \multirow{2}{*}{0.23} & \multirow{2}{*}{.82} \\
\hline & & Mujer & 7 & 1.00 & 1.00 & & \\
\hline & \multirow{2}{*}{$\mathrm{S}$} & Hombre & 9 & 1.11 & 0.60 & \multirow{2}{*}{0.48} & \multirow{2}{*}{.63} \\
\hline & & Mujer & 7 & 1.00 & 0.00 & & \\
\hline & \multirow{2}{*}{ A } & Hombre & 9 & 1.22 & 1.56 & \multirow{2}{*}{-0.25} & \multirow{2}{*}{.80} \\
\hline & & Mujer & 7 & 1.43 & 1.61 & & \\
\hline
\end{tabular}

*Significativo para $p<0.05$. Nota: $\mathrm{D}=$ Democrático, $\mathrm{S}=$ Sobreprotector, $\mathrm{P}=$ Permisivo, $\mathrm{A}=$ Autoritario.

En el análisis de los EEs de los progenitores, teniendo en cuenta el género de los menores, encontramos que, excepto en el EE Permisivo paterno, dan puntuaciones 
más altas las niñas que los niños en los EE que creen que definen a sus progenitores, aun estando en la misma categoría evaluativa en todos casos. Al realizar el análisis de correlación, encontramos diferencias significativas para $p<0,05$ en el EE Sobreprotector de los padres $(t=-2.39 ; p=0.03)$ (Tabla 6$)$.

Tabla 7. EE manifestado por los progenitores según Perfil de Estilos Educativos (PEE; Magaz y García, 1998)

\begin{tabular}{llll}
\hline & \multicolumn{1}{c}{ Estilo } & $N$ & Porcentaje \\
\hline \multirow{4}{*}{ EE del padre } & Democrático & 2 & 13.3 \\
\cline { 2 - 4 } & Sobreprotector & 5 & 33.3 \\
\cline { 2 - 4 } & Permisivo & 5 & 33.3 \\
\cline { 2 - 4 } & Autoritario & 3 & 20.0 \\
\hline \multirow{3}{*}{ EE de la madre } & Democrático & 2 & 12.5 \\
\cline { 2 - 4 } & Sobreprotector & 2 & 12.5 \\
\cline { 2 - 4 } & Permisivo & 8 & 50.0 \\
\cline { 2 - 4 } & Autoritario & 4 & 25.0 \\
\hline
\end{tabular}

Al realizar el análisis del EE manifestado por los padres a través del Perfil de Estilos Educativos (PEE), obtenemos que el EE es mayoritariamente Permisivo (33.3\%) y Sobreprotector (33.3\%) en los padres y Permisivo en madres (50.0\%). El EE Democrático es reconocido, por ambos progenitores, como el menos practicado en la educación de los menores, junto con el Sobreprotector en las madres (Tabla 7).

Tabla 8. Comparación de los EE manifestados por los progenitores y el percibido por los menores en función del sexo

\begin{tabular}{lllll}
\hline & & Manifestado por los padres & Percibido por los niños & Percibido por las niñas \\
\cline { 2 - 5 } & & $N(\%)$ & $N(\%)$ & $N(\%)$ \\
\hline \multirow{5}{*}{ EE del padre } & Democrático & $2(13.3)$ & $3(33.3)$ & $2(33.3)$ \\
\cline { 2 - 5 } & Sobreprotector & $5(33.3)$ & $0(00.0)$ & $0(00.0)$ \\
\cline { 2 - 5 } & Permisivo & $5(33,3)$ & $1(11,1)$ & $0(00,0)$ \\
\cline { 2 - 5 } & Autoritario & $3(20,0)$ & $5(55,6)$ & $4(66,7)$ \\
\hline \multirow{3}{*}{ EE de la madre } & $2(12,5)$ & $5(55,6)$ & $3(42,9)$ \\
\cline { 2 - 5 } & Democrático & $2(12,5)$ & $3(33,3)$ & $3(42,9)$ \\
\cline { 2 - 5 } & Sobreprotector & $8(50,0)$ & $0(00,0)$ & $0(00,0)$ \\
\cline { 2 - 5 } & Permisivo & $4(25,0)$ & $1(11,1)$ & $1(14,3)$ \\
\hline
\end{tabular}

Al comparar los EE manifestados por los padres y las madres con el percibido por los niños y niñas, se observa que no hay concordancia entre ellos. A pesar de los resultados obtenidos mediante el análisis de medias de puntuación, los niños piensan que sus padres ejercen un EE Democrático (33.3\%) y Autoritario (55.6\%) en la educación familiar, mientras que los padres manifiestan ejercer un EE Permisivo (33.3\%) y Sobreprotector (33.3\%). Las niñas perciben un estilo educativo del padre Autoritario (66.7\%), no contemplan ni el EE Sobreprotector ni Permisivo para sus padres.

Los niños piensan que sus madres tienen un EE Democrático (55.6\%) y Permisivo (33.3\%) en la educación familiar, mientras que las madres manifiestan ejercer un rol Permisivo (50.0\%) y Autoritario (25.0\%) en segundo lugar. El $42.9 \%$ de las niñas 
perciben un EE de las madres Democrático y Permisivo principalmente; no contemplan el EE Sobreprotector para sus madres.

Niños y niñas coinciden en no atribuir ni a sus madres ni a sus padres un $E E$ Sobreprotector, además las niñas no atribuyen tampoco un EE Permisivo a sus padres.

Los padres manifiestan ejercer un EE mayoritariamente Permisivo, que no es percibido ni por niños $(11.1 \%)$ ni por las niñas $(0 \%)$. Las madres manifiestan ejercer un rol mayoritariamente Permisivo que no que coincide con el percibido por las niñas y por los niños, donde no es contemplado (Tabla 8).

$\mathrm{Si}$ relacionamos el nivel de inadaptación de los menores con el EE manifestado por los progenitores y el percibido por los menores, observamos que no se observan diferencias significativas. No se han podido realizar pruebas post hoc entre variables de inadaptación con el EE percibido por los menores de sus padres, porque al menos un grupo tiene menos de dos casos (Tabla 9).

Tabla 9. Relación entre el EE percibido de las madres e Inadaptación de los menores, según Test Autoevaluativo de Adaptación Infantil (TAMAI; Hernández, 1990)

\begin{tabular}{|c|c|c|c|}
\hline & Estilo Educativo d & Diferencia de medias & Sig. \\
\hline \multirow{3}{*}{ Inadaptación General } & $\mathrm{D}-\mathrm{S}$ & 8.83 & .28 \\
\hline & $\mathrm{D}-\mathrm{A}$ & 4.50 & .70 \\
\hline & $\mathrm{S}-\mathrm{A}$ & 13.33 & .28 \\
\hline \multirow{3}{*}{ Inadaptación Personal } & $\mathrm{D}-\mathrm{S}$ & -4.45 & .18 \\
\hline & $\mathrm{D}-\mathrm{A}$ & -0.12 & .98 \\
\hline & $\mathrm{S}-\mathrm{A}$ & 4.33 & .38 \\
\hline \multirow{3}{*}{ Inadaptación Escolar } & $\mathrm{D}-\mathrm{S}$ & -3.41 & .30 \\
\hline & $\mathrm{D}-\mathrm{A}$ & 3.25 & .50 \\
\hline & $\mathrm{S}-\mathrm{A}$ & 6.66 & .19 \\
\hline \multirow{3}{*}{ Inadaptación Social } & $\mathrm{D}-\mathrm{S}$ & -0.96 & .71 \\
\hline & $\mathrm{D}-\mathrm{A}$ & 1.37 & .71 \\
\hline & $\mathrm{S}-\mathrm{A}$ & 2.33 & .55 \\
\hline \multirow{3}{*}{ Insatisfacción Ambiente Familiar } & $\mathrm{D}-\mathrm{S}$ & -0.37 & .61 \\
\hline & $\mathrm{D}-\mathrm{A}$ & -1.37 & .21 \\
\hline & $\mathrm{S}-\mathrm{A}$ & -1.00 & .37 \\
\hline \multirow{3}{*}{ Insatisfacción Hermanos } & $\mathrm{D}-\mathrm{S}$ & -0.50 & .59 \\
\hline & $\mathrm{D}-\mathrm{A}$ & -2.50 & .08 \\
\hline & $\mathrm{S}-\mathrm{A}$ & -2.00 & .17 \\
\hline
\end{tabular}

Nota: $\mathrm{D}=$ Democrático, $\mathrm{P}=$ Permisivo, $\mathrm{A}=$ Autoritario.

Tomando como referencia el EE que los padres manifiestan tener, se realiza estudio correlacional con las variables de Inadaptación e Insatisfacción obtenidas del TAMAI, no observándose diferencias significativas para ninguna de las comparaciones realizadas (Tabla 10). 
Tabla 10. Relación entre el EE manifestado por los padres, según Perfil de Estilos Educativos (PEE) e Inadaptación, según Test Autoevaluativo de Adaptación Infantil (TAMAI)

\begin{tabular}{|c|c|c|c|}
\hline & EE del pac & Diferencia de medias & Sig. \\
\hline \multirow{6}{*}{ Inadaptación General } & $|\mathrm{D}-\mathrm{S}|$ & 7.30 & .58 \\
\hline & $\mid \mathrm{D}-\mathrm{P}$ & 4.50 & .74 \\
\hline & $\mathrm{D}-\mathrm{A}$ & -3.17 & .83 \\
\hline & $|\mathrm{S}-\mathrm{P}|$ & -2.80 & .78 \\
\hline & $|S-A|$ & -10.47 & .38 \\
\hline & $|\mathrm{P}-\mathrm{A}|$ & -7.66 & .51 \\
\hline \multirow{6}{*}{ Inadaptación Personal } & $\mathrm{D}-\mathrm{S}$ & 3.80 & .48 \\
\hline & $\mathrm{D}-\mathrm{P}$ & 3.00 & .58 \\
\hline & $\mathrm{D}-\mathrm{A}$ & -1.33 & .82 \\
\hline & $|\mathrm{S}-\mathrm{P}|$ & -0.80 & .84 \\
\hline & $\mathrm{S}-\mathrm{A}$ & -5.13 & .28 \\
\hline & $\mathrm{P}-\mathrm{A}$ & -4.33 & .36 \\
\hline \multirow{6}{*}{ Inadaptación Escolar } & $\mathrm{D}-\mathrm{S}$ & 1.70 & .77 \\
\hline & $\mathrm{D}-\mathrm{P}$ & 1.90 & .74 \\
\hline & $\mathrm{D}-\mathrm{A}$ & 0.50 & .94 \\
\hline & $|S-P|$ & 0.20 & .96 \\
\hline & $\mathrm{S}-\mathrm{A} \mid$ & -1.20 & .81 \\
\hline & $\mathrm{P}-\mathrm{A}$ & -1.40 & .78 \\
\hline \multirow{6}{*}{ Inadaptación Social } & $\mathrm{D}-\mathrm{S}$ & 1.80 & .65 \\
\hline & $\mathrm{D}-\mathrm{P}$ & -0.40 & .92 \\
\hline & $\mathrm{D}-\mathrm{A}$ & -2.33 & .60 \\
\hline & $\mid \mathrm{S}-\mathrm{P}$ & -2.20 & .47 \\
\hline & $\mathrm{S}-\mathrm{A}$ & -4.13 & .25 \\
\hline & $|\mathrm{P}-\mathrm{A}|$ & -1.93 & .58 \\
\hline
\end{tabular}

Nota: $\mathrm{D}=$ Democrático, $\mathrm{S}=$ Sobreprotector, $\mathrm{P}=$ Permisivo, $\mathrm{A}=$ Autoritario.

Al realizar análisis del EE manifestado por los padres y su relación con las variables de insatisfacción, no observamos diferencias significativas entre los grupos analizados (Tabla 11).

Tabla 11. Relación entre el EE manifestado por los padres, según Perfil de Estilos Educativos (PEE) e Insatisfacción, según Test Autoevaluativo de Adaptación Infantil (TAMAI)

\begin{tabular}{lccc}
\hline & \multicolumn{1}{c}{ EE Padre } & Diferencia de medias & Sig. \\
\hline & $|\mathrm{D}-\mathrm{S}|$ & -0.30 & .79 \\
\cline { 2 - 4 } Insatisfacción Ambiente Familiar & $|\mathrm{D}-\mathrm{P}|$ & 0.70 & .55 \\
\cline { 2 - 4 } & $|\mathrm{D}-\mathrm{A}|$ & 0.83 & .52 \\
\cline { 2 - 4 } & $|\mathrm{S}-\mathrm{P}|$ & 1.00 & .27 \\
\cline { 2 - 4 } & $|\mathrm{S}-\mathrm{A}|$ & 1.13 & .28 \\
\hline \multirow{3}{*}{ Insatisfacción Hermanos } & $|\mathrm{P}-\mathrm{A}|$ & 0.13 & .89 \\
\cline { 2 - 4 } & $|\mathrm{D}-\mathrm{S}|$ & 0.60 & .70 \\
\cline { 2 - 4 } & $|\mathrm{D}-\mathrm{P}|$ & 0.20 & .90 \\
\cline { 2 - 4 } & $|\mathrm{D}-\mathrm{A}|$ & 1.00 & .54 \\
\cline { 2 - 4 } & $|\mathrm{S}-\mathrm{P}|$ & -0.40 & .72 \\
\cline { 2 - 4 } & $|\mathrm{S}-\mathrm{A}|$ & 0.40 & .76 \\
\hline
\end{tabular}

Nota: $\mathrm{D}=$ Democrático, $\mathrm{S}=$ Sobreprotector, $\mathrm{P}=$ Permisivo, $\mathrm{A}=$ Autoritario. 


\section{DISCUSIÓN}

Después de observar los resultados obtenidos, se comprueba que los padres manifiestan ejercer un EE Permisivo y Autoritario, siendo este último percibido también en primer lugar por los menores. El EE predominante manifestado por los padres y madres es el Permisivo, EE que es percibido por los menores en sus padres, aunque en la elección individual se aprecie que eligen como EE de los padres el Autoritario en primera opción, y Democrático en segunda. Para las madres, también aparece coincidencia en la elección, habiéndose considerado el número de menores que eligen cada EE, siendo en este caso los EE Democrático y Sobreprotector los elegidos.

Aunque no se han encontrado diferencias significativas entre niños y niñas a la hora de percibir el EE de sus padres y madres, el EE predominante percibido por las niñas y por los niños de sus padres es el autoritario. Cuando clasifican el EE de las madres, niños y niñas están de acuerdo en que el principal EE es el Autoritario, aunque las niñas presenten mayores puntuaciones. Según estudio realizado por Oliva et al. (2007) y García et al. (2011) sí que encuentran diferencias significativas en la percepción entre niños y niñas en el EE paterno, siendo las niñas más sensibles a las diferencias entre ambos progenitores. Probablemente, en nuestro estudio, el que no se encuentren diferencias significativas entre niños y niñas a la hora de percibir el EE del padre ni de la madre, se deba al tamaño reducido de la muestra.

También es importante resaltar que la muestra de menores además de ser escasa en tamaño, no es proporcional en relación a la edad, y es menor la proporción de niñas que de niños. Las niñas presentan edades más avanzadas respecto de los niños, por lo que existen otros factores a tener en cuenta como puede ser la aceleración del desarrollo madurativo en éstas.

Se observa, que no hay concordancia entre los EE manifestados por los padres y el percibido por niños y niñas. Los niños perciben el ejercicio de un rol autoritario y democrático en la educación familiar por parte de sus padres, mientras que los padres manifiestan ejercer un rol permisivo y sobreprotector. Las niñas no contemplan ni el rol sobreprotector ni el permisivo para sus padres, éstas perciben un EE del padre mayoritariamente autoritario.

Los niños perciben el ejercicio de un rol democrático y sobreprotector de sus madres en la educación familiar, mientras que las madres manifiestan ejercer un rol permisivo principalmente. Las niñas no perciben en sus madres un rol sobreprotector y democrático. Fuentes et al. (2001) también encontraron baja concordancia entre la información aportada por los padres y sus hijos.

Los menores, coinciden en no atribuir ni a sus madres ni a sus padres un $E E$ Sobreprotector, además las niñas no atribuyen tampoco un estilo permisivo a sus padres. Los padres manifiestan ejercer un rol mayoritariamente permisivo y sobreprotector, que 
no es percibido ni por niños ni por las niñas, y las madres manifiestan ejercer un rol mayoritariamente permisivo y autoritario que no coincide con el percibido por los menores.

Los padres piensan que consienten a sus hijos, y los hijos perciben este EE como Democrático y Autoritario para niños y niñas; ni las niñas ni los niños se sienten sobreprotegidos por parte de sus progenitores. De todo esto, podríamos deducir la existencia de desacuerdo por parte de las niñas y de los niños hacia el EE ejercido por el padre.

Con estos resultados, podríamos llegar a pensar que niños y niñas consideran a su padre como una figura de autoridad, aunque los niños también le dan un rango Democrático. La figura de autoridad estaría ejercida por el padre, siendo las madres más permisivas que los padres, según modelo patriarcal.

Respecto del nivel de Inadaptación de los menores y EE (Figura 1), se puede observar que:

Los menores presentan niveles altos de Inadaptación Escolar cuando el EE de la madre es Permisivo. Afirmación que difiere con la manifestada por algunos autores como Cerezo et al. (2011) que manifestaban que los menores cuyos padres son Democráticos y Permisivos obtienen mayores logros escolares. Otros autores como Fernández y Salvador (1994), Steinberg (1989), Lamborn et al. (1991) y Domínguez y Carton (1997) relacionan negativamente el EE Permisivo de los padres con el rendimiento escolar de sus hijos/as, y sobre todo en menores en situación de riesgo social (Platone 2007). Bornstein y Bornstein (2010) relacionan el ejercicio de un EE Democrático en los padres con el crecimiento intelectual en el menor.

El EE Democrático de la madre parece influir negativamente en el nivel de satisfacción con hermanos. Aparecen diferencias en los niveles de insatisfacción con los hermanos en los EE Permisivo y Democrático de la madre, con resultado mayor en el EE Democrático. Por lo que podríamos deducir que en un clima de autoridad, afecto y flexibilidad por parte de la figura materna, los menores manifiestan más insatisfacción con sus hermanos.

La Inadaptación Personal del niño es mayor en los EE Autoritario y Democrático del padre. López-Soler et al. (2009) relacionaban positivamente el EE Autoritario con la Inadaptación Personal y Social en niños y niñas, y otros como Lamborn et al. (1991), Domínguez y Carton (1997) y García-Linares et al. (2002) relacionan el estilo democrático con altos niveles de autoestima.

Cuando los menores perciben un EE Permisivo del padre se produce mayor Insatisfacción con los Hermanos. La percepción de un EE Democrático del padre influye en el grado de Inadaptación Escolar y Social. El índice de Insatisfacción con el Ambiente Familiar e Insatisfacción con los Hermanos es menor con un EE Democrático que con los demás, siendo éste más representativo en chicos que en chicas. Autores 
como Rollán et al. (2011) llegaron también a la conclusión de que los EE Permisivo, Punitivo, Restrictivo, Despreocupado y Perfeccionista se relacionan con la Inadaptación Familiar, y el EE Democrático con la adaptación familiar de los/las hijos/as.

Figura 1. Esquema relacional entre EEs y áreas de inadaptación

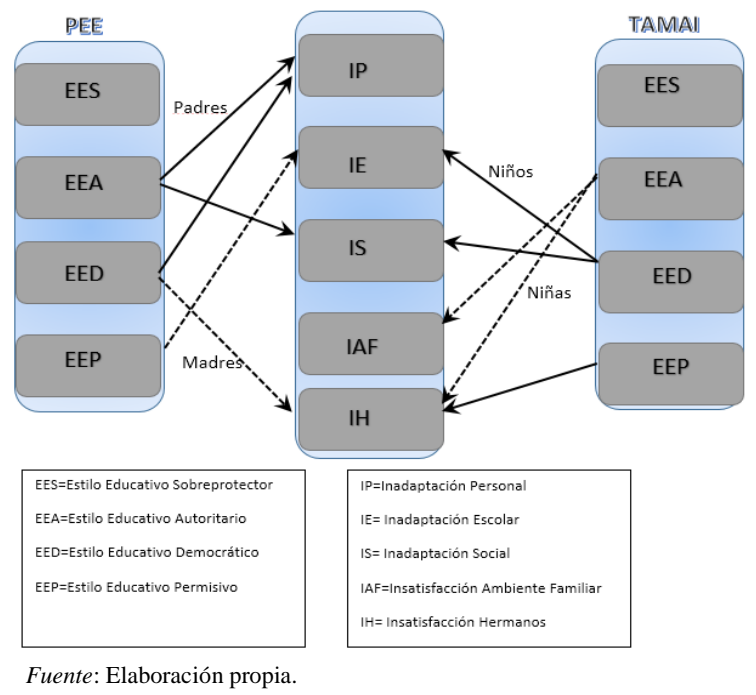

Según los resultados de nuestro estudio y tras análisis de qué tipo de socialización es idóneo según ajuste psicosocial, observamos que diferentes autores como García y Gracia (2010), Ríos (1973), Rodríguez (1986), Alonso y Román (2005), García-Linares et al. (2002) y García et al. (2011) determinan que el estilo familiar democrático se relaciona positivamente con nivel de adaptación de los menores, siendo dicho estilo es el más adecuado en poblaciones normalizadas, ya que facilita una mejor adaptación (Steinberg, 2001).

Una de las limitaciones con la que nos encontramos en este estudio, es la dificultad para extrapolar estos resultados al resto de la población. Debemos tener en cuenta que la población objeto de estudio es una población peculiar en relación a la presentación de características comunes que la caracterizan; es por tanto una muestra bastante homogénea. Las familias en situación de riesgo social, presentan factores de riesgo e indicadores de maltrato que han sido determinados con anterioridad a la inclusión en el Programa de tratamiento a familias con menores (Dirección General de Infancia y Familia, 2007).

Los EEs no suelen ser modelos puros, sino mixtos, se suelen solapar y varían con el desarrollo del menor y no suelen ser estables a lo largo del tiempo. Pueden cambiar, por lo que es importante analizar el EE en el contexto en el que tiene lugar, en 
la realidad de cada familia y momento evolutivo en el que se encuentre el menor. En nuestro estudio se utilizan instrumentos de evaluación que determinan EEs considerados puros.

Otra de las dificultades con las que nos encontramos en este estudio a la hora de sacar conclusiones, es la de determinar si el EE que se evalúa es el que realmente se está llevando a cabo, ya que el Test Autoevaluativo de Adaptación Infantil (TAMAI; Hernández, 1990) pone de manifiesto la percepción de los menores y el Perfil de EEs (PEE; Magaz y García, 1998) la percepción de los padres y puede que no se realice una valoración objetiva.

Nos encontramos igualmente con el tamaño reducido de la muestra, por lo que sería necesaria la elaboración de más estudios de investigación con esta población para poder encontrar más evidencia al respecto. Los resultados obtenidos sobre estudio de la relación entre el Estilo Educativo parental y nivel de adaptación de los menores de esta muestra pueden servir de orientación para la elaboración de futuros programas de intervención con este tipo de colectivos y plantear iniciativas de mejora a los programas de intervención psicosocial existentes, con la finalidad de marcar pautas sobre cuáles son los EEs que más favorecen a los menores en situación de riesgo social.

Sería igualmente importante en futuras investigaciones ampliar la muestra de estudio para comparar la consistencia entre los distintos EEs con las diferentes subescalas de inadaptación, así como la utilización de otros instrumentos de medida para poder comparar los resultados obtenidos tanto por el Test Autoevaluativo de Adaptación Infantil (TAMAI; Hernández, 1990) como con el Perfil de EEs (PEE; Magaz y García, 1998). En base a las limitaciones de nuestro estudio y teniendo en cuenta las aportaciones realizadas por diferentes autores, se pone en evidencia la necesidad de trabajar con el modelo de familia que favorezca el desarrollo de la personalidad de los menores, que estimule sus capacidades y le ofrezca seguridad y aprendizaje.

Resaltar la importancia de la utilización de un EE en el que exista equilibrio entre el afecto y la autoridad, que ofrezca clima de seguridad al menor, que tenga presente en todo momento sus necesidades de aprendizaje y le ofrezcan las oportunidades para asumir retos en función de su desarrollo madurativo. Sería un EE, que exige de los adultos habilidades necesarias para afrontar la educación diaria del menor. La adopción de un EE parental que ejerce su autoridad con responsabilidad, afecto y flexibilidad es el más beneficioso para el crecimiento emocional, moral e intelectual del niño (Bornstein y Bornstein, 2010).

\section{CONCLUSIONES}

Según los resultados de nuestro estudio, en los menores se aprecia un nivel de inadaptación general, sobre todo en inadaptación personal, escolar e insatisfacción con el 
ambiente familiar, donde la existencia de discrepancias educativas podría ser una de las causas de esta inadaptación en los mismos. Existen también diferencias de percepción del EE entre padres y menores, por lo que es difícil asociar, sin margen de error, la inadaptación con cualquiera de los EEs. En la esfera de percepción de los menores, ellos relacionan la Inadaptación Escolar con un EE Permisivo de su madre, se sienten insatisfechos con sus hermanos cuando la madre lleva a cabo un EE Democrático. Cuando el EE es contemplado desde el punto de vista de los padres, aparecen factores de inadaptación personal asociados a un EE Autoritario y de satisfacción familiar (único factor positivo) asociado a un EE democrático.

\section{REFERENCIAS}

Alonso, J. y Román, J.M. (2005). Prácticas educativas familiares y autoconcepto. Estudio con niños y niñas de 3,4 y 5 años. Departamento de psicología. Tesis doctoral no publicada. Universidad de Valladolid.

Ato, E., Galián, M.D. y Huéscar, E. (2007). Relaciones entre EEs, temperamento y ajuste social en la infancia: una revisión. Anales de Psicología, 23(1), 45-53.

Bornstein, L. y Bornstein, M.H. (2010). EEs Parentales y el Desarrollo Social del niño. En R.E. Tremblay, R.G. Barr, RDeV. Peters y M. Boivin, (Eds.), Enciclopedia sobre el desarrollo de la primera infancia (pp.1-4). Montreal: En línea.

Caplan, S., Henderson, C., Henderson, J. y Fleming, D. (2002). Socio-emotional factor contributing to adjustment among early-entrance college students, Gifted Child Quarterly, 46(2), 124-134.

Castejón, J.L. y Pérez, A.M., (1998). Un modelo casual explicativo de las variables psicosociales en el rendimiento académico. Revista Bordón, 50(2), 171-185.

Cerezo, M.T., Casanova, P.F., De la Torre, M.J. y Carpio, M. (2011). EEs paternos y estrategias de aprendizaje en alumnos de educación secundaria. European Journal of Education and Psychology, 4(1), 51-61.

De Barros, D.P., Primi, R., Miguel, F.K., Almeida, L.S. y Oliveira, E.P. (2010). Metaphor creation: A measure of creativity or intelligence. European Journal of Education and Psychology, 3(1), 103-115.

De la Rubia, J.M. y Ortíz, H. (2011). Modelos predictivos de la conducta disocial por sexos en dos colonias populares. Salud Mental, 34(3), 227-235.

Dirección General de Infancia y Familia, 2007. Programa de Tratamiento a familias con menores. Junta de Andalucía. Consejería para la Igualdad y Bienestar Social.

Domínguez, M. y Cartons, J. (1997). The relations hip between sejf-actualization and parenting style. Journal of social Behavoir and Personality 12(4), 1093-1100.

Fernández, S. y Salvador, F. (1994). La familia ante el fracaso escolar. Educadores, 36(169), 7-22.

Ferreira, A.I., Martínez, L.F. y Guisande, M.A. (2009). Risky behavior, personality traits and road accidents among university students. European Journal of Education and Psychology, 2(2), 79-98.

Fuentes, M.J., Bersabé, R. y Motrico, E. (2001). Prácticas educativas paternas y problemas internalizantes y externalizantes en adolescentes españoles. Psicothema, 13(4), 678-684.

Fuentes, MC., García, J.F., Gracia, E. y Lila, M. (2011). Autoconcepto y ajuste social en la adolescencia. Psicothema, 23(1), 7-11. 
García, F. y Gracia, E. (2010) ¿Qué estilo de socialización parental es el idóneo en España? Un estudio con niños y adolescentes de 10 a 14 años. Infancia y Aprendizaje, 33(3), 365384.

García, M.C., Cerezo, M.T., De la Torre, M.J., Carpio, M. y Casanova, P. (2011). Prácticas educativas paternas y problemas internalizantes y externalizantes en adolescentes españoles. Psicothema, 23(4), 654-659.

García-Linares, M.C., Pelegrina, S. y Lendínez, J. (2002). EEs de los padres y competencia social de los adolescentes. Anuario de Psicología, 33(1), 80-95.

Gázquez, J.J., Pérez-Fuentes, M.C. y Carrión, J.J. (2009). Factores familiares, individuales y sociales que influyen en el origen y desarrollo de la violencia escolar según nuestros mayores. Revista Galego-Portuguesa de Psicoloxía y Educación, 18(2), 97-105.

Gázquez, J.J., Pérez-Fuentes, M.C., Ruíz, M.I., Miras, F. y Vicente, F. (2006). Estrategias de aprendizaje de enseñanza secundaria obligatoria y su relación con la autoestima. International Journal of Psychology and Psychological Therapy, 6(1), 51-62.

Goñi, E. e Infante, G. (2010). Actividad físico-deportiva, autoconcepto físico y satisfacción con la vida. European Journal of Education and Psychology, 3(2), 199-208.

Hernández, P. (1990). TAMAI. Test Autoevaluativo Multifactorial de Adaptación Infantil. Madrid: TEA.

Lamborgn, S.D. (1991). Patterns of competence and adjustment among adolescents from. Child Development, 62(5), 1049-1065.

Llorente, M. (1990). Fracaso escolar y origen social. Tesina no publicada. Universidad Pontificia de Salamanca.

López-Soler, C., Puerto, J.C., López-Pina, J.A. y Prieto, M. (2009). Percepción de los EEs parentales e inadaptación en menores pediátricos. Anales de Psicología, 25(1), 70-77.

Maccoby, E.E. (1992). The role of parents in the socialization of children: An historical overview. Developmental Psychology, 28, 1006-1017.

Magaz, A. y García, E.M. (1998). PEE. Perfil de EEs. Madrid: Albor-Cohs.

Marchesi, A. y Martín, E. (2002). Evaluación de la educación secundaria. Fotografía de una etapa polémica. Instituto IDEA, Madrid: SM.

Martínez, R. (2004). Evaluación e intervención educativa en el campo familiar. Revista Española de Orientación y Psicopedagogía, 15(1), 89-104.

Martínez-Otero, V. (1997). Los adolescentes ante el estudio. Causas y consecuencias del rendimiento académico. Madrid: Fundamentos.

Oliva, A., Parra, A., Sánchez, I. y López, F. (2007). EEs materno y paterno: evaluación y relación con el ajuste adolescente. Anales de Psicología, 23(1), 23-31.

Pichardo, M.C. (1998). Influencia del EE educativo de los padres y del clima social familiar en la adolescencia temprana y media. Tesis Doctoral no publicada. Universidad de Granada.

Platone, M.N. (2007). Condiciones familiares y maltrato infantil. Pensamiento Psicológico, 3(8), 53-60.

Ríos, J.A. (1973). Fracaso escolar y vida familiar. Madrid: Marsiega.

Rodríguez, E. (1986). Vida familiar y fracaso escolar: comparación de grupos extremos de rendimiento. Tesina no publicada. Universidad Pontificia de Salamanca.

Rollán, C., Masedra, P., Sánchez, J.C., Ruíz, C. Presa, M. y León, I. (2011). De la investigación intercultural a la adaptación preventiva de la familia: mejora de Autoconcepto y adaptación social, EEs de padres y adaptación familiar en hijos. Educación y Futuro Digital, 38(1), 5-6.

Salguero, J.M., Fernández-Berrocal, P., Ruiz-Aranda, D., Castillo, R. y Palomera, R. (2011). Inteligencia emocional y ajuste psicosocial en la adolescencia: El papel de la percepción emocional. European Journal of Education and Psychology, 4(2), 143-152. 
Santiago, M.J., Otero, J.M., Castro, C. y Villardefrancos, E. (2008). Occupational stress in secondary school teachers: examining the role of students' disruptive behaviour and/or attitudes and the perceived difficulty in conflict management. European Journal of Education and Psychology, 1(1), 39-50.

Steinberg, I. (1989). Autoritative parenting psychosocial maturity and academic success avnong adolescents. Child Developement, 60(6), 1424-1436.

Steinberg, I. (2001). We know some things: Adolescent-parent relationships in retrospect and prospect. Journal of Adolescence, 11(1), 1-20.

Torio, S., Peña, J.V. e Inda, M. (2008). Estilos de educación familiar. Psicothema, 20(1), 62-70.

Valverde, J. (1988). El proceso de Inadaptación Social. Madrid: Popular.

Recibido: 20 de septiembre de 2013

Recepción Modificaciones: 8 de noviembre de 2013

Aceptado: 11 de noviembre de 2013 